PROCEEDINGS OF THE

AMERICAN MATHEMATICAL SOCIETY

Volume 130, Number 2, Pages 433-442

S 0002-9939(01)06281-5

Article electronically published on August 7, 2001

\title{
RELAXATION AND CONVEXITY OF FUNCTIONALS WITH POINTWISE NONLOCALITY
}

\author{
EUGENE STEPANOV
}

(Communicated by Jonathan M. Borwein)

\begin{abstract}
It is shown that the relaxation of the integral functional involving argument deviations

$$
I(u):=\int_{\Omega} f\left(x,\left\{u_{i}\left(g_{i j}(x)\right)\right\}_{i, j=1}^{k, l}\right) d \mu_{\Omega}(x),
$$

in weak topology of a Lebesgue space $\left(L^{p}\left(\Theta, \mu_{\Theta}\right)\right)^{k}$ (where $\left(\Omega, \Sigma(\Omega), \mu_{\Omega}\right)$ and $\left(\Theta, \Sigma(\Theta), \mu_{\Theta}\right)$ are standard measure spaces, the latter with nonatomic measure), coincides with its convexification whenever the matrix of measurable functions $g_{i j}: \Omega \rightarrow \Theta$ satisfies the special condition, called unifiability, which can be regarded as collective nonergodicity or commensurability property, and is automatically satisfied only if $k=l=1$. If, however, either $k>1$ or $l>1$, then it is shown that as opposed to the classical case without argument deviations, for nonunifiable function matrix $\left\{g_{i j}\right\}$ one can always construct an integrand $f$ so that the functional $I$ itself is already weakly lower semicontinuous but not convex.
\end{abstract}

\section{INTRODUCTION}

Let $\left(\Omega, \Sigma(\Omega), \mu_{\Omega}\right)$ and $\left(\Theta, \Sigma(\Theta), \mu_{\Theta}\right)$ be standard measure spaces (i.e. $\Omega$ and $\Theta$ Polish spaces, $\Sigma(\Omega)$ and $\Sigma(\Theta)$ completions of the respective Borel $\sigma$-algebrae with respect to the finite Borel measures $\mu_{\Omega}$ and $\mu_{\Theta}$ ). The reference to the particular $\sigma$-algebra and measure will be omitted for the sake of brevity, whenever this cannot lead to confusion. The measure $\mu_{\Theta}$ is further supposed to be nonatomic.

Define the functional $I: L_{k}^{p} \equiv\left(L^{p}(\Theta)\right)^{k} \rightarrow \mathbb{R} \cup\{+\infty\} \equiv \overline{\mathbb{R}}$ by the relationship

$$
I(u):=\int_{\Omega} f\left(x,\left\{u_{i}\left(g_{i j}(x)\right)\right\}_{i, j=1}^{k, l}\right) d \mu_{\Omega}(x),
$$

where $u:=\left(u_{1}, \ldots, u_{k}\right)$ is a vector with components $u_{i} \in L^{p}(\Theta), i=1, \ldots, k, f$ : $\Omega \times \mathbb{R}^{k l} \rightarrow \overline{\mathbb{R}}$ is an integrand (i.e. a $\Sigma(\Omega) \otimes \mathcal{B}^{k l}$ measurable function, $\mathcal{B}^{k l}$ standing for the Borel $\sigma$-algebra of $\mathbb{R}^{k l}$ ), while $g_{i j}: \Omega \rightarrow \Theta, i=1, \ldots, k, j=1, \ldots, l$, are measurable functions satisfying

$$
e \subset \Theta, \mu_{\Theta}(e)=0 \Rightarrow \mu_{\Omega}\left(g_{i j}^{-1}(e)\right)=0 .
$$

Note that the latter condition is necessary to define correctly the compositions $u_{i} \circ g_{i j}$ for $u_{i} \in L^{p}(\Theta)$ (or just for $u_{i}$ being classes of $\mu_{\Theta}$-a.e. equal measurable functions). Thus we will always assume it is satisfied.

Received by the editors June 15, 2000.

2000 Mathematics Subject Classification. Primary 49J45; Secondary 47B37, 47H30, 49J25.

(C)2001 American Mathematical Society 
The primary source of such functionals is provided by optimal control theory. In fact, an optimal control problem for differential equations with deviating argument can be easily reduced by introducing auxiliary variables as in 1, 2, to the purely variational problem of minimizing a functional of the type (II) with $k>1$. It is worth noting however that there are also other applications of such functionals even in the "scalar" case $k=1$, mainly related to functional differential equations with variational structure (see [3] and the references therein).

This paper studies the relationship between convexity and weak lower semicontinuity of the above functionals. In particular, we study when the relaxation of the latter in the weak topology $s c^{-}\left(w-L_{k}^{p}\right) I$ (i.e. the greatest weakly lower semicontinuous functional less than $I$ ) coincides with its convexification $I^{* *}$ (i.e. the greatest convex and strongly lower semicontinuous functional less than $I$ ). It is shown that the situation in general changes drastically with respect to the well-studied case $k=l=1, \Omega=\Theta$ with $g_{11}(x)=x$, sometimes referred to as "local". The wellknown results of A. Ioffe show that in this case the convexity of the functional (and even, in an appropriate sense, that of the integrand) is necessary for the functional to be weakly lower semicontinuous (see, e.g., [5, 6, 1]). This is however strictly related to the fact that in the definition of the functional one then has the local (namely, Nemytskiǐ) operator of $u$ under the integration sign. In the presence of just "pointwise" nonlocality given by the functions $g_{i j}$, as we show, convexity in general is no more necessary for the functional studied to be weakly lower semicontinuous. We provide, moreover, a precise condition on the functions $g_{i j}$ in order that the relaxation of these functionals coincide with their convexifications. This condition is satisfied automatically only if $k=l=1$ (whatever the function $g_{11}$ is), while in general it is a special extension to function matrices of the notion of nonergodicity of a single function. It provides a very strong relationship between functions in each row and a rather weak relationship between functions in each column.

\section{UNIFIABILITY OF FUNCTIONS AND ORBITAL CONDITIONS}

We will call a measurable (with respect to $\Sigma(\Theta)$ ) function $\gamma: \Theta \rightarrow \mathbb{R}$ a unifier of the vector row of functions $\left(g_{1}, \ldots, g_{l}\right), g_{i}: \Omega \rightarrow \Theta, i=1, \ldots, l$, if

$$
\gamma\left(g_{1}(x)\right)=\ldots=\gamma\left(g_{l}(x)\right)
$$

for $\mu_{\Omega}$-a.e. $x \in \Omega$. Note that the set of unifiers for a given vector row of functions is closed with respect to convergence in measure, hence also with respect to pointwise convergence.

Definition 1. The vector row of functions $\left(g_{1}, \ldots, g_{l}\right), g_{i}: \Omega \rightarrow \Theta, i=1, \ldots, l$, is called unifiable if it admits a unifier $\gamma: \Theta \rightarrow \mathbb{R}$ satisfying

$$
\mu_{\Theta}\left(\gamma^{-1}(y)\right)=0
$$

for all $y \in \mathbb{R}$.

We emphasize that we are deliberately speaking of vector rows of functions (and not just of sets). In fact, although it changes nothing in the scalar case (the order of functions in the row has clearly no importance), the distinction will be obvious in the vectorial case.

It is easy to see that not every vector row of functions is unifiable. 
Example 1. Let $\left(\Theta, \Sigma(\Theta), \mu_{\Theta}\right)=\left(\Omega, \Sigma(\Omega), \mu_{\Omega}\right)$. Then the simplest example of a nonunifiable row of functions is given by a pair $g_{1}:=\mathrm{id}_{\Omega}$ (the identity map) and $g_{2}$ : $\Omega \rightarrow \Omega$ any ergodic map. In fact, the ergodicity of $g_{2}$ means that the only function $\gamma$ satisfying $\gamma(x)=\gamma\left(g_{2}(x)\right)$ for $\mu_{\Omega}$-a.e. $x \in \Omega$ is a constant [4]. A classical particular example of the ergodic map is the rotation of the circle $\Omega=S^{1}$ (equipped with the one-dimensional Hausdorff measure) by the angle $2 \pi \alpha$ where $\alpha$ is irrational. It can also be stated in terms of transformation of the interval $\Omega=(0,1)$ (with the usual Lebesgue measure) as $g(x)=\{x+\alpha\}$ where $\{\cdot\}$ stands for the fractional part function.

In view of the above example the unifiability can be understood as the natural extension of nonergodicity property to collections of functions. Note, for instance, that the vector row of rotations of the circle $S^{1}$ by the angles $2 \pi \alpha_{i}, i=1, \ldots, l$, is unifiable if and only if the angles are commensurable (i.e. they relate with each other as rational numbers).

Now we try to study the unifiability condition from another point of view. For this purpose for each $x \in \Omega$ define the orbit $O(x) \subset \Theta$ by

$$
O(x):=\bigcup_{j \in \mathbb{N}} O_{j}(x)
$$

where

$$
\begin{aligned}
& O_{0}(x):=\{x\} \\
& O_{1}(x):=\left\{y \in \Theta: g_{i}^{-1}(y) \cap g_{j}^{-1}(x) \neq \emptyset \text { for some } i \neq j\right\} \\
& O_{j}(x):=O_{1}\left(O_{j-1}(x)\right) .
\end{aligned}
$$

Observe that $O$ defines an equivalence relation $x \sim y \Leftrightarrow x \in O(y)$.

We will say that a set $e \subset \Theta$ is $O$-invariant if $O(e)=e$. It is easy to note that $O$ invariant subsets form a $\sigma$-algebra. By $\Sigma_{O}$ we denote the $\sigma$-algebra of $O$-invariant sets belonging to $\Sigma(\Theta)$. A real valued function on $\Theta$ will be called $O$-measurable if it is measurable with respect to $\Sigma_{O}$.

Recall that an atom of a $\sigma$-algebra $\Sigma^{\prime} \subset \Sigma(\Theta)$ is is a set $E \in \Sigma^{\prime}$ with $\mu_{\Theta}(E)>0$ such that for every $e \subset E, e \in \Sigma^{\prime}$ one has either $\mu_{\Theta}(e)=0$ or $\mu_{\Theta}(e)=\mu_{\Theta}(E)$. $\Sigma^{\prime}$ is said to be nonatomic if it contains no atoms. With these notions at hand we are able to prove the following statement about the relationship between unifiability of $\left(g_{1}, \ldots, g_{l}\right)$ and nonatomicity of $\Sigma_{O}$.

Lemma 1. If the vector row of functions $\left(g_{1}, \ldots, g_{l}\right)$ is unifiable, then $\Sigma_{O}$ contains a nonatomic $\sigma$-subalgebra.

Proof. Let the vector row $\left(g_{1}, \ldots, g_{l}\right)$ be unifiable by means of a unifier $\gamma: \Theta \rightarrow \mathbb{R}$ satisfying (3). We will show that $\gamma^{-1}(\mathcal{B}) \subset \Sigma_{O}$, where $\mathcal{B}$ stands for the $\sigma$-algebra of Borel subsets of $\Omega$, is nonatomic. Suppose the contrary, namely that the latter has an atom $E$. The set $D \in \mathcal{B}, \gamma^{-1}(D)=E$ is necessarily more than countable, and hence by the theorem of Kuratowski [7] there is a measurable one-to-one map $j: D \rightarrow[0,1)$. Now consider the map $\gamma^{\prime}:=j \circ \gamma: E \rightarrow[0,1)$. For each $\nu \in \mathbb{N}$ let

$$
B_{\nu}^{i}:=\left[(i-1) / 2^{\nu}, i / 2^{\nu}\right), \quad i=1, \ldots, 2^{\nu} .
$$

Since $\bigsqcup_{j} B_{\nu}^{j}=[0,1)$, then there is a $i^{\prime}=i^{\prime}(\nu)$ such that $\mu_{\Theta}\left(\gamma^{\prime}-1\left(B_{\nu}^{i^{\prime}(\nu)}\right)\right)=\mu_{\Theta}(E)$. 
Clearly, $B_{\nu+1}^{i^{\prime}(\nu+1)} \subset B_{\nu}^{i^{\prime}(\nu)}$ and

$$
\omega_{0}:=\bigcap_{\nu} B_{\nu}^{i^{\prime}(\nu)}
$$

consists of no more than one point. Therefore, we have an obvious contradiction

$$
0=\mu_{\Theta}\left(\gamma^{\prime}-1\left(\omega_{0}\right)\right)=\mu_{\Theta}\left(\bigcap_{\nu} \gamma^{\prime-1}\left(B_{\nu}^{i^{\prime}(\nu)}\right)\right)=\mu_{\Theta}(E),
$$

where the first equality comes from (3).

\section{Relaxation and Convexity: Scalar case}

We first concentrate on the scalar case $k=1$, and show that the phenomenon announced in the introduction reveals itself already in this setting, and hence does not have any special "vectorial" nature. To abbreviate the notation, we omit everywhere the unnecessary index 1, i.e. we write $g_{i}$ instead of $g_{1 i}, u$ instead of $u_{1}$ and $L^{p}$ instead of $L_{1}^{p}$. The functional $I: L^{p} \rightarrow \overline{\mathbb{R}}$ defined by (1) is then reduced to

$$
I(u)=\int_{\Omega} f\left(x, u\left(g_{1}(x)\right), \ldots, u\left(g_{l}(x)\right)\right) d x .
$$

We are now in a position to prove that its relaxation in the weak topology of $L^{p}$ is convex, and hence coincides with its convexification, provided the vector row $\left(g_{1}, \ldots, g_{l}\right)$ is unifiable.

Theorem 1. Let $1 \leq p \leq+\infty$. If the vector row of functions $\left(g_{1}, \ldots, g_{l}\right)$ is unifiable, then

$$
s c^{-}\left(w-L^{p}\right) I(u)=I^{* *}(u),
$$

where in the case $p=+\infty$ the relaxation is meant in the $*$-weak topology.

We emphasize that according to the definition of unifiability in the case $k=1$ this theorem implies no extra condition on $g_{1}$.

Proof. The proof is identical for $1<p<+\infty$ and $p=+\infty$ modulo changing the words "weak topology" to "*-weak topology" in the latter case. To show the claim, it is enough to prove that $s c^{-}\left(w-L^{p}\right) I$ is convex. Thus consider the arbitrary $\left\{u_{1}, u_{2}\right\} \subset L^{p}$ and let the sequences $\left\{u_{\nu}^{1}\right\},\left\{u_{\nu}^{2}\right\}$ be such that

$$
u_{\nu}^{i} \rightarrow u_{i} \text { weakly in } L^{p}, \text { while } s c^{-}\left(w-L^{p}\right) I\left(u_{i}\right)=\lim _{\nu} I\left(u_{\nu}^{i}\right), \quad i=1,2 .
$$

Pick up a countable dense set $\left\{p_{j}\right\} \subset L^{p^{\prime}}, 1 / p^{\prime}+1 / p=1$, and fix an arbitrary $\lambda \in(0,1)$. Since the vector row $\left(g_{1}, \ldots, g_{l}\right)$ is unifiable, according to Lemma 1 there is a nonatomic $\sigma$-algebra $\Sigma^{\prime} \subset \Sigma_{O}$. 
Consider for each fixed $\nu \in \mathbb{N}$ the following systems of equations with respect to $e_{\nu} \in \Sigma^{\prime}$ :

$$
\left\{\begin{array}{c}
\int_{e_{\nu}} p_{j}(y) u_{\nu}^{1}(y) d \mu_{\Theta}(y)=\lambda \int_{\Theta} p_{j}(y) u_{\nu}^{1}(y) d \mu_{\Theta}(y) \\
\int_{\Theta\left(e_{\nu}\right.} p_{j}(y) u_{\nu}^{2}(y) d \mu_{\Theta}(y)=(1-\lambda) \int_{\Theta} p_{j}(y) u_{\nu}^{2}(y) d \mu_{\Theta}(y) \\
\int_{g_{1}^{-1}\left(e_{\nu}\right)} f\left(x, u_{\nu}^{1}\left(g_{1}(x)\right), \ldots, u_{\nu}^{1}\left(g_{l}(x)\right)\right) d \mu_{\Omega}(x) \\
=\lambda \int_{\Omega} f\left(x, u_{\nu}^{1}\left(g_{1}(x)\right), \ldots, u_{\nu}^{1}\left(g_{l}(x)\right)\right) d \mu_{\Omega}(x) \\
\int_{\Omega \backslash g_{1}^{-1}\left(e_{\nu}\right)} f\left(x, u_{\nu}^{2}\left(g_{1}(x)\right), \ldots, u_{\nu}^{2}\left(g_{l}(x)\right)\right) d \mu_{\Omega}(x) \\
=(1-\lambda) \int_{\Omega} f\left(x, u_{\nu}^{2}\left(g_{1}(x)\right), \ldots, u_{\nu}^{2}\left(g_{l}(x)\right)\right) d \mu_{\Omega}(x)
\end{array}\right.
$$

where $j=1, \ldots, \nu$. To solve this system, introduce the vector measure $\mu^{\nu}: \Sigma^{\prime} \rightarrow$ $\mathbb{R}^{2 \nu+2}$ by the following formulae:

$$
\begin{aligned}
\mu_{j}^{\nu}(e) & :=\int_{e} p_{j}(y) u_{\nu}^{1}(y) d \mu_{\Theta}(y), \quad j=1, \ldots, \nu, \\
\mu_{\nu+j}^{\nu}(e) & :=\int_{e} p_{j}(y) u_{\nu}^{2}(y) d \mu_{\Theta}(y), \quad j=1, \ldots, \nu, \\
\mu_{2 \nu+1}^{\nu}(e) & :=\int_{g_{1}^{-1}(e)} f\left(x, u_{\nu}^{1}\left(g_{1}(x)\right), \ldots, u_{\nu}^{1}\left(g_{l}(x)\right)\right) d \mu_{\Omega}(x), \\
\mu_{2 \nu+2}^{\nu}(e) & :=\int_{g_{1}^{-1}(e)} f\left(x, u_{\nu}^{2}\left(g_{1}(x)\right), \ldots, u_{\nu}^{2}\left(g_{l}(x)\right)\right) d \mu_{\Omega}(x) .
\end{aligned}
$$

The system of equations (5) can then be written as

$$
\mu^{\nu}\left(e_{\nu}\right)=\lambda \mu^{\nu}(\Theta)
$$

It is clear that it admits a solution thanks to the Lyapunov theorem on convexity of the range of a vector measure (note that $\mu_{2 \nu+1}^{\nu}<<\mu_{\Theta}, \mu_{2 \nu+2}^{\nu}<<\mu_{\Theta}$ and hence are nonatomic). Therefore the first and second equations in (5) imply

$$
\chi_{e_{\nu}} u_{\nu}^{1}+\chi_{\Theta \backslash e_{\nu}} u_{\nu}^{2} \rightarrow \lambda u_{1}+(1-\lambda) u_{2}
$$

weakly in $L^{p}$, and hence, by definition of $s c^{-}\left(w-L^{p}\right) I$ with the help of the third and the fourth equations of (5), we obtain

$$
\begin{aligned}
s c^{-}\left(w-L^{p}\right) I & \left(\lambda u_{1}+(1-\lambda) u_{2}\right) \leq \liminf _{\nu} I\left(\chi_{e_{\nu}} u_{\nu}^{1}+\chi_{\Theta \backslash e_{\nu}} u_{\nu}^{2}\right) \\
& =\lambda \lim _{\nu} I\left(u_{\nu}^{1}\right)+(1-\lambda) \lim _{\nu} I\left(u_{\nu}^{2}\right) \\
& =\lambda s c^{-}\left(w-L^{p}\right) I\left(u_{1}\right)+(1-\lambda) s c^{-}\left(w-L^{p}\right) I\left(u_{2}\right),
\end{aligned}
$$

concluding the proof for the case $p \neq 1$. If $p=1$, one should reiterate the above arguments taking $\left\{p_{j}\right\}$ to be a countable dense subset in the space of continuous functions, which gives (6) in the weak sense of measures. It is then enough to observe that in fact (6) also holds in the sense of weak convergence in $L^{1}$, because the sequence $\left\{\chi_{e_{\nu}} u_{\nu}^{1}+\chi_{\Theta \backslash e_{\nu}} u_{\nu}^{2}\right\}$ is equi-integrable, since the sequences $\left\{u_{\nu}^{i}\right\}, i=1,2$, are as well.

Corollary 1. If the set of functions $g_{1}, \ldots, g_{l}: \Omega \rightarrow \Theta$ is unifiable, then for the functional I defined by (4) to be weakly lower semicontinuous over $L^{p}(\Theta), 1 \leq p<$ 
$+\infty$ (resp. *-weakly lower semicontinuous in $L^{\infty}$ ), it is necessary and sufficient that it be convex and lower semicontinuous in the strong topology.

Now we pass to the more difficult question, namely, what happens when the vector row $\left(g_{1}, \ldots, g_{l}\right)$ is not unifiable. We show that in this case in general convexity has nothing to do with weak lower semicontinuity of the respective functionals. The basic idea for this proof is provided by the example below.

Example 2. Let $\left(g_{1}, g_{2}\right)$ be a nonunifiable function pair given by Example 1 namely, $g_{1}:=\operatorname{id}_{\Omega}$ and $g_{2}: \Omega \rightarrow \Omega$ any ergodic map. Define $f: \mathbb{R}^{2} \rightarrow \overline{\mathbb{R}}$ by

$$
f\left(y_{1}, y_{2}\right):=\left\{\begin{aligned}
0, & y_{1}=y_{2} \geq 1 \text { or } y_{1}=y_{2} \leq 0 \\
+\infty, & \text { elsewhere }
\end{aligned}\right.
$$

Then for the functional

$$
I(u):=\int_{\Omega} f\left(u\left(g_{1}(x)\right), u\left(g_{2}(x)\right)\right) d \mu_{\Omega}(x)
$$

one has $I(u)=0$, only if $u \circ g_{1}=u \circ g_{2} \mu_{\Omega}$-a.e. in $\Omega$. However, this can happen only if $u=$ const in view of ergodicity of $g_{2}$. Hence

$$
I(u)=\left\{\begin{aligned}
0, & u=\text { const } \geq 1 \text { or } u=\text { const } \leq 0 \\
+\infty, & \text { elsewhere }
\end{aligned}\right.
$$

which implies that $I$ is weakly lower semicontinuous, i.e. $I=s c^{-}\left(w-L^{p}\right) I$, but not convex, namely, $I \neq I^{* *}$.

We start now with the following technical auxiliary statement which will be of utmost importance for our construction.

Lemma 2. If the vector row of functions $\left(g_{1}, \ldots, g_{l}\right), g_{i}: \Omega \rightarrow \Theta, i=1, \ldots, l$, is not unifiable, then there exists a measurable set $\hat{\Theta} \subset \Theta$ such that every unifier is constant over $\hat{\Theta}$.

Proof. Step 1. For each measurable $\gamma: \Theta \rightarrow \mathbb{R}$ we set

$$
M_{\gamma}:=\sup _{s \in \mathbb{R}} \mu_{\Theta}\left(\gamma^{-1}(s)\right) .
$$

Clearly, in view of nonunifiability of the functions $g_{1}, \ldots, g_{l}$ one has $M_{\gamma}>0$ for each unifier $\gamma$. We prove that

$$
M:=\inf _{\gamma} M_{\gamma}>0
$$

where inf is taken over all the unifiers $\gamma$ of $\left(g_{1}, \ldots, g_{l}\right)$.

Suppose the contrary, i.e. that there exists a sequence of unifiers $\gamma^{\nu}$ such that $M_{\gamma^{\nu}} \rightarrow 0$ when $\nu \rightarrow \infty$. Obviously, for every $\gamma^{\nu}$ there exist no more than a countable number of points $s_{j}^{\nu} \in \mathbb{R}$ such that $\mu_{\Theta}\left(\gamma^{-1}\left(s_{j}^{\nu}\right)\right)>0$. Denote

$$
\begin{aligned}
C_{j}^{\nu} & :=\left(\gamma^{\nu}\right)^{-1}\left(s_{j}^{\nu}\right), & V^{\nu} & :=\Theta \backslash \bigcup_{j} C_{j}^{\nu}, \\
V & :=\bigcup_{\nu} V^{\nu}, & C & :=\Theta \backslash V=\bigcap_{\nu} \bigcup_{j} C_{j}^{\nu}, \\
W^{\nu} & :=V^{\nu} \backslash \bigcup_{l=1}^{\nu-1} V^{l}, & C^{j_{1} j_{2} \ldots j_{\nu}} & :=C \cap\left(\bigcap_{\nu} C_{j_{\nu}}^{\nu}\right) .
\end{aligned}
$$

For every $\nu \in \mathbb{N}$ we define the function $\hat{\gamma}^{\nu}: \Theta \rightarrow \mathbb{R}$ by setting

$$
\hat{\gamma}^{\nu}(x):= \begin{cases}\gamma^{l}(x), & x \in W^{l}, \quad l \in \mathbb{N}, \\ c^{\nu}(x), & x \in C,\end{cases}
$$


where the function $c^{\nu}: C \rightarrow \mathbb{R}$ is defined by

$$
c^{\nu}(x):=1+1 / 2^{j_{1}}+1 / 2^{j_{1}+j_{2}}+\ldots+1 / 2^{j_{1}+j_{2}+\ldots+j_{\nu}}, \quad x \in C^{j_{1} j_{2} \ldots j_{\nu}} \cap C .
$$

It is clear that $\hat{\gamma}^{\nu}$ is a unifier, since all the sets introduced above are $O$-invariant.

Now we notice that the sequence $\hat{\gamma}^{\nu}$ is nondecreasing and bounded, so it has a pointwise limit $\hat{\gamma}$, which is also a unifier. We prove that $\hat{\gamma}$ satisfies (3), thus contradicting the nonunifiability assumption and proving (7). Indeed, if it is not so, then there exists a point $s \in \mathbb{R}$ such that $\mu_{\Theta}\left(\hat{\gamma}^{-1}(s)\right)>0$. Note that then by construction $\hat{\gamma}^{-1}(s) \subset C$, and for some $\nu \in \mathbb{N}$ such that $M_{\gamma^{\nu}}<\mu_{\Theta}\left(\hat{\gamma}^{-1}(s)\right)$ we have $\hat{\gamma}^{-1}(s)$ covering several $C_{j}^{\nu} \cap C$. That means that $\hat{\gamma}$ cannot take a single value over $\hat{\gamma}^{-1}(s)$ and leads to a contradiction.

Step 2. Consider now a sequence of unifiers $\gamma^{\nu}$ such that $M_{\gamma^{\nu}} \rightarrow M$ when $\nu \rightarrow \infty$. We preserve the notation from the Step 1 . For every $\nu \in \mathbb{N}$ we have

$$
\mu_{\Theta}\left(C^{j_{1} j_{2} \ldots j_{\nu}}\right) \geq M
$$

for some $j_{1}, j_{2}, \ldots, j_{\nu} \in \mathbb{N}$. Indeed, otherwise we could have constructed a unifier $\hat{\gamma}^{\nu}$ with $M_{\hat{\gamma}^{\nu}}<M$. Moreover, for every $\varepsilon>0$ one has

$$
\mu_{\Theta}\left(C^{j_{1} j_{2} \ldots j_{\nu}}\right) \leq M+\varepsilon
$$

for all sufficiently large $\nu \in \mathbb{N}$. Combining (8) and (9), one concludes the existence of at least one and no more than a finite number of measurable $O$-invariant sets $\hat{\Theta}_{j}, \mu_{\Theta}\left(\hat{\Theta}_{j}\right)=M$, such that for every $\nu \in \mathbb{N}$ one has $\hat{\Theta}_{j} \subset C^{j_{1} j_{2} \ldots j_{\nu}}$ for some $j_{1}, j_{2}, \ldots, j_{\nu} \in \mathbb{N}$. Besides, since all $\Theta_{j}$ are $O$-invariant, we can construct a unifier $\hat{\gamma}$ such that $\hat{\Theta}_{j}=\hat{\gamma}^{-1}\left(s_{j}\right)$ for some points $s_{j} \in \mathbb{R}$.

Note that among the sets $\hat{\Theta}_{j}$ there exists at least one set $\hat{\Theta}$ such that every unifier $\gamma$ takes a single value over $\hat{\Theta}$. Indeed, otherwise we could have divided each $\hat{\Theta}_{j}$ into several parts of positive measure and construct a unifier $\tilde{\gamma}$ with $M_{\tilde{\gamma}}<M$.

Now we are able to prove that in fact the unifiability condition is unavoidable both in Theorem 1 and in Corollary 1 . Namely, the following assertion is valid.

Theorem 2. If the set of functions $g_{1}, \ldots, g_{l}: \Omega \rightarrow \Theta$ is not unifiable, then there exists an integrand $f: \Theta \times \mathbb{R}^{k} \rightarrow \overline{\mathbb{R}}$ such that the respective functional $I$ defined by (4) is weakly lower semicontinuous over $L^{p}$ (resp. *-weakly lower semicontinuous in $L^{\infty}$ ) and not convex.

Proof. According to Lemma 2 there exists a set $\hat{\Theta} \in \Sigma(\Theta)$ such that every unifier of $\left(g_{1}, \ldots, g_{l}\right)$ takes a single value over $\hat{\Theta}$. Consider the set $G$ of all unifiers $\gamma \in L^{p}$ such that $|\gamma(x)| \geq 1$ for $\mu_{\Theta}$-a.e. $x \in \hat{\Theta}$. We prove that $G$ is closed in the weak topology of $L^{p}$. In fact, consider an arbitrary sequence $\gamma^{\nu} \in G$ such that $\gamma^{\nu} \rightarrow \gamma$ weakly. Clearly, $\gamma$ is still a unifier, since it is $O$-measurable. It remains to show that $|\gamma(x)| \geq 1$ for $\mu_{\Theta}$-a.e. $x \in \hat{\Theta}$. Since $\gamma^{\nu}(x) \equiv c^{\nu}$ and $\gamma(x) \equiv c$ for $\mu_{\Theta}$-a.e. $x \in \hat{\Theta}$, while $\left|c^{\nu}\right| \geq 1$, we have $c^{\nu} \rightarrow c$, and hence $|c| \geq 1$ as well. Thus $G$ is closed in the weak topology of $L^{p}$, but is clearly nonconvex.

Now we construct an integrand $f$ by setting

$f(x, y):=\left\{\begin{aligned} 0, & g_{i}(x) \in \hat{\Theta} \text { and there exists } \alpha \in \mathbb{R}: \text { all } y_{i}=\alpha, \text { while }|\alpha|>1, \\ +\infty, & \text { elsewhere. }\end{aligned}\right.$ 
It is clear that the functional $I$ defined by (4) is then

$$
I(u)= \begin{cases}0, & u \in G, \\ +\infty, & u \notin G,\end{cases}
$$

and hence is lower semicontinuous in the weak topology of $L^{p}(\Theta)$ but not convex.

Note that the assertions we proved tell only that unifiability condition for the row $\left(g_{1}, \ldots, g_{l}\right)$ is necessary for the relaxation of the functional (4) in weak topology of $L^{p}$ to coincide with its convexification for a generic integrand $f$. Nevertheless, for a particular integrand it may happen that the respective relaxation coincides with the convexification of the functional even without the unifiability requirement. For instance, by modifying slightly the proof of Theorem 1 one concludes that when

$$
f\left(x, y_{1}, \ldots, y_{l}\right)=f_{1}\left(x, y_{1}, \ldots, y_{m}\right)+f_{2}\left(x, y_{m+1}, \ldots, y_{l}\right)
$$

for some $m \in\{1, \ldots, l\}$, then the statement of Theorem 1 remains valid only under the condition that each of the two vector rows of functions $\left(g_{1}, \ldots, g_{m}\right)$ and $\left(g_{m+1}, \ldots, g_{l}\right)$ be unifiable, which is obviously much less restrictive than the unifiability of the whole row $\left(g_{1}, \ldots, g_{l}\right)$.

\section{Relaxation and COnvexity: Vectorial CASE}

Now consider the general vectorial setting (1). We now extend Definition 1 to the case of function matrices $\left\{g_{i j}\right\}_{i, j=1}^{k, l}$.

Definition 2. A vector row of measurable (with respect to $\Sigma(\Theta)$ ) functions

$$
\gamma=\left(\gamma_{1}, \ldots, \gamma_{k}\right), \quad \gamma_{i}: \Theta \rightarrow \mathbb{R}, i=1, \ldots, k
$$

is said to be a unifier of the function matrix $\left\{g_{i j}\right\}_{i, j=1}^{k, l}$ if all the functions $\gamma_{i} \circ g_{i j}$ : $\Omega \rightarrow \mathbb{R}$ coincide $\mu_{\Omega}$-a.e. over $\Omega$.

Definition 3. The function matrix $\left\{g_{i j}\right\}_{i, j=1}^{k, l}$ is called unifiable if it admits such a unifier $\left(\gamma_{1}, \ldots, \gamma_{k}\right)$ that

$$
\mu_{\Theta}\left(\gamma_{i}^{-1}(y)\right)=0, \quad i=1, \ldots, k
$$

for all $y \in \mathbb{R}$.

Let us make some remarks. First, if $k=1$, then the function matrix $\left\{g_{i j}\right\}_{i, j=1}^{k, l}$ reduces to a row and we are in the case of Definition 1. But if instead $l=1, k>1$, then the above function matrix is a vector column. The unifiability condition in this case is actually much less restrictive than for vector rows. In fact, just injectivity of each of the functions in the column would suffice for the unifiability of the whole column, which is clearly not the case for unifiability of rows. Namely, the following trivial statement holds.

Proposition 1. A vector column of injective functions $\left(g_{1}, \ldots, g_{k}\right)^{T}$ (where $T$ stands for transposition, to distinguish rows from columns) is unifiable.

Proof. Since all the functions $g_{i}$ satisfy (2), up to restricting them to the Borel subset of full measure in $\Omega$, we may assume that the respective inverses $g_{i}^{-1}$ satisfy (2). Assuming without loss of generality $\mu_{\Omega}(\Omega)=1$, we consider the measure preserving one-to-one map $\theta: \Omega \rightarrow[0,1]$ (assuming $[0,1]$ is equipped with the standard Lebesgue measure). Hence it is enough to take $\gamma_{i}:=\theta \circ g_{i}^{-1}, i=1, \ldots, k$. 
However, still not all vector columns of functions are unifiable, as the example below shows.

Example 3. Let $\Theta=\Omega=(0,1)$. Set

$$
\begin{aligned}
& g_{1}(x):=1 / 2+1 / 4 \cos 2 \pi x \\
& g_{2}(x):=1 / 2+1 / 4 \cos 2 \pi(x-\alpha / 2),
\end{aligned}
$$

where $\alpha$ is some irrational number. Suppose that there exists a unifier $\left(\gamma_{1}, \gamma_{2}\right)$ of the vector column $\left(g_{1}, g_{2}\right)^{T}$ and consider the function $g=\gamma_{1} \circ g_{1}=\gamma_{2} \circ g_{2}$. Let us take an arbitrary point $x \in \Omega$. First, since $g_{1}(x)=g_{1}(1-x)$, we have $g(x)=g(1-x)$. Second, since $g_{2}(1-x)=g_{2}(\{1-(1-x)+\alpha\})=g_{2}(\{x+\alpha\})$, we have $g(1-x)=g(\{x+\alpha\})$. Summing up, we obtain $g(x)=g(\{x+\alpha\})$, which means that $g$ is a constant function, and hence $\gamma_{1}$ and $\gamma_{2}$ are constant over $g_{1}(\Omega)$ and $g_{1}(\Omega)$ respectively.

At last is seems worth mentioning that according to our definition a matrix consisting of a single function (i.e. $k=l=1$ ) is always unifiable.

Now we are in a position to extend Theorems 1 and 2 to the general vectorial case.

Theorem 3. Let $1 \leq p \leq+\infty$. If the function matrix $\left\{g_{i j}\right\}_{i, j=1}^{k, l}$ is unifiable, then for the functional $I$ defined by (1) one has

$$
s c^{-}\left(w-L_{k}^{p}\right) I(u)=I^{* *}(u),
$$

where in the case $p=+\infty$ the relaxation is meant in the $*$-weak topology.

If the function matrix $\left\{g_{i j}\right\}_{i, j=1}^{k, l}$ is not unifiable, then there is an integrand $f$ : $\Omega \times \mathbb{R}^{k l} \rightarrow \overline{\mathbb{R}}$ such that the respective functional I defined by (1) is weakly lower semicontinuous over $L_{k}^{p}$ (resp. *-weakly lower semicontinuous in $L_{k}^{\infty}$ ) and not convex.

Proof. Let $\Theta^{\prime}:=\{1, \ldots, k\} \times \Theta$ be equipped with the product $\sigma$-algebra $2^{\{1, \ldots, k\}} \otimes$ $\Sigma(\Theta)$, the measure being the product of the uniform measure in $\{1, \ldots, k\}$ and $\mu_{\Theta}$. Obviously, the linear operator $T: L_{k}^{p} \rightarrow L^{p}\left(\Theta^{\prime}\right)$ defined by

$$
(T u)(i, x))=u_{i}(x), \quad i=1, \ldots, k, x \in \Theta
$$

is an isomorphism. Thus the weak lower semicontinuity of $I$ over $L_{k}^{p}$ is equivalent to that of $I \circ T^{-1}$ over $L^{p}\left(\Theta^{\prime}\right)$. But the latter functional is of the type (41) (i.e. we are again in the scalar case) involving the set of deviations $\bar{g}_{i j}: \Omega \rightarrow \Theta^{\prime}$ defined by

$$
\bar{g}_{i j}(x):=\left(i, g_{i j}(x)\right) .
$$

Assemble them in a single row of $k \times l$ elements. Clearly, unifiability of this row is equivalent to that of the original function matrix $\left\{g_{i j}\right\}_{i, j=1}^{k, l}$, and therefore the conclusion follows from the results of the previous section.

\section{ACKNOWLEDGMENTS}

The author is grateful to L. De Pascale for his valuable comments. 


\section{REFERENCES}

[1] G. Buttazzo. Semicontinuity, Relaxation and Integral Representation in the Calculus of Variations, volume 207 of Pitman research notes in mathematics. Longman Scientific, Harlow, 1989. MR 91c: 49002

[2] G. Buttazzo, M.E. Drakhlin, L. Freddi, and E. Stepanov. Homogenization of optimal control problems for functional differential equations with deviating argument. J. Optim. Theory and Appl., 93(1):103-119, 1997. MR 98d:49015

[3] M.E. Drakhlin, E. Litsyn, and E. Stepanov. Variational methods for a class of nonlocal functionals. Computers and Math. with Appl., 37(4/5):79-100, 1999. MR 2000a:49006

[4] I.I. Gikhman and A.V. Skorokhod. The Theory of Stochastic Processes. "Nauka", Moscow, 1975. in Russian. English transl.: Springer-Verlag, Berlin, 1980. MR 82k:60005

[5] A. Ioffe. On lower semicontinuity of integral functionals. I. SIAM J. Control and Optimization, 15(4):521-538, 1977. MR 58:30610a

[6] A. Ioffe. On lower semicontinuity of integral functionals. II. SIAM J. Control and Optimization, 15(6):991-1000, 1977. MR 58:30610b

[7] C. Kuratowski. Topologie, volume 1. Państwowe Wydawnictwo Naukowe, Warszawa, 1958. in French. MR 19:873d

Dipartimento di Matematica, Universitá di Pisa, via Buonarroti 2, 56127 Pisa, italy

E-mail address: stepanov@cibs.sns.it 